\title{
The use of topical corticoids in oral pathology
}

\author{
Miguel-Ángel González-Moles
}

Full Professor of Oral Medicine, University of Granada

\author{
Correspondence: \\ Department of Stomatology. \\ School of Dentistry. \\ Campus de Cartuja sn, \\ 18071 - Granada, Spain \\ magonzal@ugr.es
}

Received: 13/10/2009

Accepted: 06/03/2010

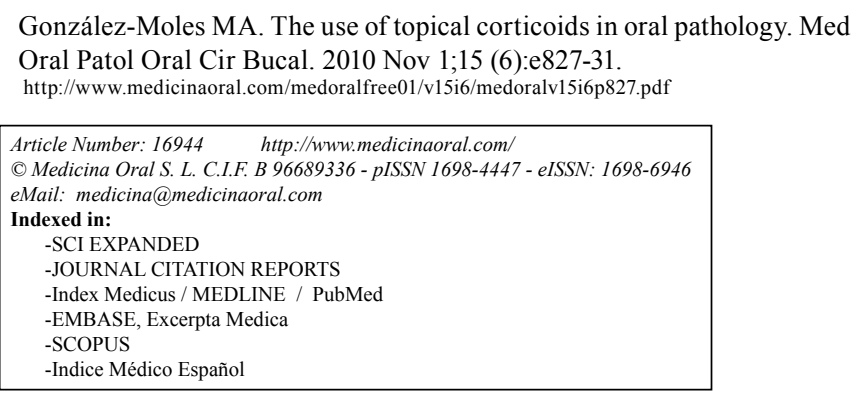

\begin{abstract}
This review examines the most important aspects of the use of topical corticosteroids in oral pathology. We provide a detailed analysis of the most common types of corticosteroids used, their methods of application and the clinical data that must be managed in order to monitor the effectiveness of the treatment. We also analyze the correctable causes of treatment failure and the possible adverse effects that may occur after applying these drugs. All of this is achieved by conducting a critical review of these concepts.
\end{abstract}

Key words: Topical corticosteroids, oral mucosa.

\section{Introduction}

Topical corticosteroids (TC) are some of the most common drugs used in oral pathology for treating atrophicerosive lesions that affect the mucosa. This type of lesion is rather significant given that it often bleeds and is painful; sometimes it is chronic or has a high tendency to reappear and to interfere with very important activities such as eating, drinking, speaking or associating with other people, making this type of lesion very debilitating for the patient. The seriousness of these lesions increases in such cases where the gums are affected, given that the bleeding, the inability to brush the teeth properly, bad breath and deterioration of the physical appearance of the patient may contribute to his or her social isolation. Severe erosive lesions of the oral mucosa have classically been treated with systemic corticosteroids, although the adverse effects associated with the use of these drugs have conditioned the fre- quent prescription of topical corticosteroids for treating these pathologies. However, there are few studies on the subject.

This review includes the most important aspects pertaining to the use of TCs in oral pathology, particularly the application instructions, the most common types of $\mathrm{TCs}$, their formulation and the treatment procedures, as well as the possible adverse effects associated with their use.

\section{Instructions for using TCs in oral medicine}

The use of a TC for treating various pathologies of the oral mucosa that may respond to these drugs must adhere to one of the following criteria $(1,2)$ :

- Use of a short course of TCs (2-6 days). This will be especially indicated in oral mucosal diseases that have a natural tendency towards spontaneous resolution, such as non-complex recurrent aphthous stomatitis (RAS), or 
drug-induced ulcers, once we have removed or replaced the causative drug. The use of this regimen enables reducing the natural duration of the process as well as its symptomatology.

- Use of a prolonged course of TCs of an unpredictable duration. Diseases eligible for receiving this type of treatment are chronic or very recurrent erosive lesions, such as some cases of oral lichen planus (OLP), mucous membrane pemphigoid (MMP), or some forms of complex recurrent aphthous stomatitis where outbreaks occur seamlessly, thereby making it possible for the patient to suffer permanent lesions, or to be free of lesions only for short periods of time. In order to thus reduce the percentage of recurrent cases, patients must be informed about the possibility of having to be subjected to prolonged treatments, and also about the inadvisability of discontinuing the treatment without the express consent of the doctor.

- Use of TCs along with a short course (5-6 days) of systemic corticosteroids. This regimen applies fundamentally to the treatment of very severe erosive lesions in which the use of a systemic corticoid will enable a quick resolution of the symptoms, in order to then continue monitoring the process using a topical corticosteroid.

- Finally, some diseases are not susceptible to be treated with TCs. In general, the involvement of multiple mucosas and the presence of elevated titers of circulating lesion antibodies discourage the use of topical treatments, given that the existence of multiple targets and lesion antibodies present in the plasma lesion indicate a generalized disorder of the body which will require a systemic treatment. Examples of these diseases are pemphigus vulgaris and certain forms of MMP with involvement of multiple mucous membranes.

The basic rule for managing TCs in oral pathology should be to use a TC of a strength that adapts to the seriousness of the clinical symptoms, prescribed at the lowest concentration possible, (respecting the effectiveness of the treatment) and in a form that minimizes as much as possible the mucosa area exposed to the drug. It should be kept in mind that treatment with TCs is symptomatic (non-curative). Thus, the suppression of the drug or discontinuing the treatment by the patient is often followed by a recurrence of the symptoms of the disease. Patients should be clearly informed about this aspect (1-3).

\section{TCs commonly used in oral pathology}

Although there are different types of TCs with different strengths, the clinician should be familiar with a limited number of TCs. In the scientific literature on the topic, the corticosteroids that have received greater attention and on which there is more experience are clobetasol propionate $(\mathrm{CP})$, fluocinonide and triamcinolone acetonide.
Clobetasol propionate (CP) is considered to be a superpotent corticosteroid, and according to some authors, its use has enabled restricting the use of systemic corticosteroids in oral pathology, given that many of the severe erosive lesions that required systemic administration of drugs for control of such lesions can now be controlled with CP (4). The articles published on the topic recommend using the drug at a concentration that ranges between $0.025 \%$ and $0.05 \%$, two to three times per day, and for a period of three to five minutes each application. By following this regimen, the $\mathrm{CP}$ achieves rapid control of the lesions with a low number of applications each day, also enabling longer periods free of lesions once the treatment is discontinued $(5,6)$. Our group has reported excellent results with the use of $\mathrm{CP}$, both in an aqueous solution as well as in adhesive forms $(4,7)$. In our opinion, this drug should be reserved for the treatment of severe atrophic-erosive lesions of the oral mucosa, considering those to include multiple or extensive lesions that cause intense pain, impeding or making it difficult to swallow or speak $(1,2)$ (Fig. 1).

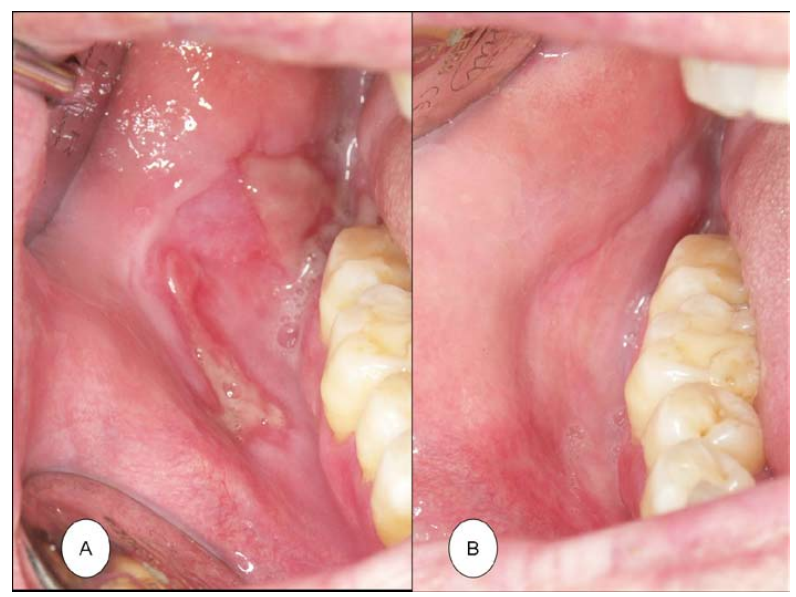

Fig. 1. A) Patient with MMP, presenting severe erosive lesions in the cheek mucosa. Lesions are also present in other parts of the oral cavity. B) Complete disappearance of the lesions after using $0.05 \%$ $\mathrm{CP}$ in aqueous solution.

Fluocinonide is considered to be a moderate-high potency corticosteroid. Studies on the subject recommend the use of the drug at concentrations ranging between $0.025 \%$ and $0.05 \%$, it being necessary to apply the medication 5-10 times per day and for 3-5 minutes in order to obtain results (6). It is a milder TC than clobetasol propionate and is less effective in reducing the pain that results from the lesions. When using the medication at a $0.025 \%$ concentration, a complete resolution of the lesions is only achieved in $25 \%$ of the patients with OLP $(5,6)$.

Finally, triamcinolone acetonide is one of the most commonly used TCs in oral pathology. It is considered to 
be an average strength TC. Studies on the subject recommend concentrations that range between $0.05 \%$ and $0.5 \%$, it being necessary to apply the medicine 3-10 times per day, and for a period of 3 to 5 minutes each time in order to achieve results. Some important authors in this field do not consider the drug to be very useful, presenting as the main drawback the fact that several applications are required each day, which poses a significant inconvenience for the patient, making it difficult to comply with the treatment $(3,8)$. However, studies published with the use of triamcinolone acetonide have recently yielded contradictory results, having reported positive effects in the treatment of OLP (9). In our opinion, triamcinolone acetonide should be used for the treatment of mild erosive lesions, considering such lesions to include those that occur with few symptoms and which are small in size $(1,2)$ (Fig. 2).

Other less explored TCs are fluticasone propionate, betamethasone sodium phosphate or momethasone. One group has reported very positive results with the use of momethasone, and minimal adverse effects $(10,11)$.

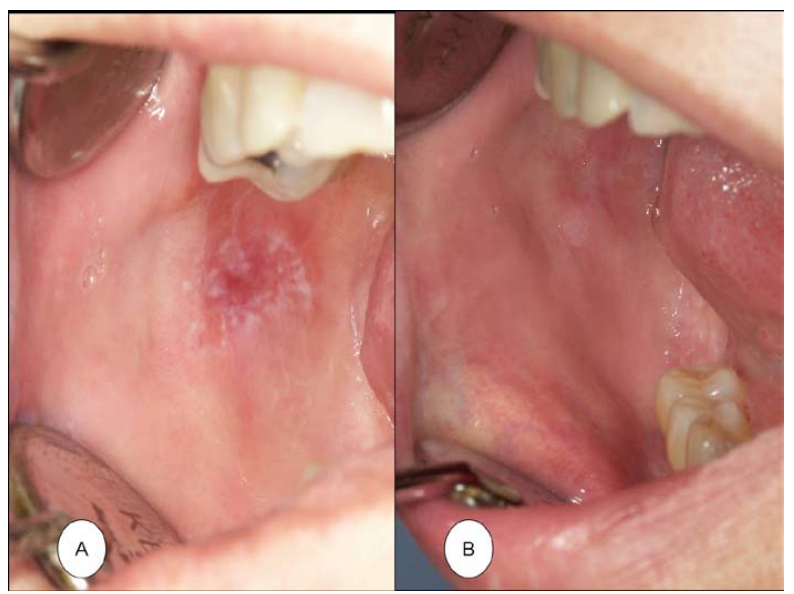

Fig. 2. A) Patient presenting oral lichenoid lesion (OLL) This small atrophic and slightly erosive lesion only appeared in the right cheek mucosa. Its low degree of seriousness and its accessible location prompted the use of $0.4 \%$ triamcinolone acetonide ointment in orabase. B) Complete disappearance of the lesion after treatment.

\section{Factors that affect the effectiveness of treatment with TCs in oral pathology}

The intrinsic strength of the drug, which is conditioned by the halogenation of its main ingredient (cortisol), is one of the factors that influence the effectiveness of treatment with TCs. Obviously, the use of a stronger TC (CP) implies a greater likelihood of achieving a complete disappearance of the lesions.

The esterification of the cortisol, which increases the penetration of the TC through the mucous membranes, also increases the effectiveness of the treatment.
The increase of the concentration of the drug, up to a certain point, is a factor which increases the possibility of achieving adequate results. However, it has been verified that there is a certain level after which increasing the concentration does not lead to an increase in the effectiveness of the TC.

One of the factors that play the largest role in determining the success of the treatment with a TC is the amount of time that the drug comes into direct contact with the lesion. This is not surprising, given that the basic requirement in a topical treatment is that the drug comes into contact with the lesions for the amount of time prescribed by the doctor. The amount of time that the drug comes into contact with the lesion depends fundamentally on the means used for applying the TC. The means most often used in oral pathology are adhesive ointments and aqueous solutions. Among adhesive ointments, the orabase ointment is one of the most commonly used. It is a commercial formula that does not contain analgesics or antibiotics. Gels are rarely used due to the possibility of causing pain after being applied. The ointments do not have adhesive properties, although they are frequently used as a usual base of the main ingredient and as a means for locating it in other forms. Finally, it was recently reported that adhesive pastes for dentures have very good adherent qualities and can be used successfully for applying TC $(1,2)$.

When TC is used in an adhesive form, it is recommended that a small amount be applied in the target area, to not eat or drink for 20 minutes and to not rub the medicine against the lesion, so as to avoid irritating the area. Some authors recommend supplying the medicine to the patient in small containers with the amount necessary for each application. At the end of the treatment, the patient should return the empty and full containers to the doctor, which in turn enables monitoring the patient's compliance with the treatment (8).

The main inconvenience raised by the use of TC in adhesive form is that it is difficult to perfectly control the amount of time that the medicine comes into contact with the lesion, given that according to some studies, the majority of the medicine quickly spreads away from the location where it was initially applied $(85-90 \%$ loss). In addition, it is difficult to apply the medicine on deep lesions or to ensure that large lesions come into equal contact with the TC. On the other hand, some patients report intolerance to the granular texture of the medicine. In our opinion, a TC should be supplied in an adhesive form when treating small, isolated lesions, or when there are very few lesions and they are easily accessible, or when the lesions are located on the gums or the palate onto which the medicine may be applied using a customized tray, thereby allowing perfect control of the amount of time that the medicine must come into contact with the lesion. Patients who wear a full set of 
dentures can use the dentures as a tray for applying the medicine $(1,2)$.

TCs can also be applied in aqueous solutions. The dentist must advise the patient of the advantage of not using hydroalcoholic solutions that would irremediably cause intense pain upon contact with atrophic-erosive lesions, and would make it difficult to comply with the treatment. The basic advantages of using aqueous solutions are the ability to have perfect control of the time that the medicine comes into contact with the lesion, contact of the medicine with all of the oral lesions regardless of their depth or how large, and it is easier to administer the corticosteroid when in the form of an aqueous solution as opposed to an adhesive form. The main drawbacks of the aqueous solution are that all of the mucosa, whether diseased or healthy, comes into contact with the medicine, thereby increasing systemic absorption of the medicine and the possibility of involuntary ingestion. We believe that the aqueous solutions should be used for treating large and deep lesions $(1,2)$.

\section{Objectives of treatment with TCs in oral patho- $\log \mathbf{y}$}

Given that treatment with TCs only achieves a symptomatic and not a curative effect, the fundamental objective of the treatment with TC in oral pathology is to ensure that the symptoms of the illness disappear, restoring the patient's ability to ingest food, speak, carry out proper oral hygiene and a normal relationship with the people of his or her environment. In essence, the main objective of the treatment is to suppress or reduce the pain. This is also usually accompanied by a disappearance or a considerable reduction of the size of the ulcerations. Changing the appearance of the white lesions in OLP or ensuring that the atrophy disappears are not the objectives of the treatment. The white lesions that accompany OLP have an unpredictable response to corticosteroids, and in some cases, the white color even increases in intensity. Similarly, the area that responds worse to treatment with TCs is usually the mucosal atrophy, which manifests as a fragile and delicate mucosa with a reddened appearance $(4,7)$. Usually, after applying TC and even after having completely resolved the pain, there are areas in the oral cavity which still present characteristics of atrophic mucosa. These atrophic lesions are usually not painful and, in our experience, it is very difficult to full restore the normal appearance of the oral mucosa. We have reported that the conversion of a painful erosive lesion into a non-painful atrophic lesion should be considered a success in the treatment with TCs. The non-objective parameters of the treatment should be taken into consideration in order to avoid unnecessary overdoses of the drug or confusion regarding the effectiveness of the treatment $(1,2)$.

\section{Treatment failures}

Despite the fact that correct instructions and use of TCs in oral pathology reveal a high degree of success in the treatment, a small percentage of patients do not respond adequately to these drugs. In our study (4), $6 \%$ of the patients with severe erosive lesions of the oral mucosa did not respond to treatment with $\mathrm{CP}$. Before attributing the treatment failure to an actual lack of response to the $\mathrm{TC}$, some of the possible causes of false treatment failures should be analyzed:

- The incorrect diagnosis and thus the incorrect indication of a TC is a cause of false treatment failure.

- Prescription of a TC of an inadequate strength, in an inadequate form or for a reduced amount of time is a common cause of false treatment failure. In our opinion, this is especially frequent with the use of triamcinolone acetonide in adhesive forms when prescribed by inexperienced dentists for the treatment of severe erosive lesions of the oral mucosa.

- Poor cooperation on the part of the patient is a cause of false lack of response to the treatment.

- Finally, some adverse affects or abnormal reactions of the mucosa following the treatment may also be confused with a lack of response. The appearance of erythematous candidiasis, with reddening and pain, as an adverse effect of the TC can be interpreted as a worsening of the symptoms of the illness. It may also occur with the increase of white lesions in patients with OLP or with the persistence of non-painful atrophic lesions following the use of TCs $(1,2)$.

\section{Adverse effects of treatment with TCs in oral pathology}

- Local adverse effects

Among the local adverse effects of treatment with TCs, there is the appearance of oral candidiasis in $25 \%-55 \%$ of the patients. The frequency of presenting candidiasis as a complication of the treatment is directly related with the use of stronger TCs in aqueous forms, for prolonged periods of times, and at high concentrations. The status of a healthy carrier (healthy patients who develop more than 50 colony-forming units in an oral mucosa culture) also affects the frequency of occurrence. Oral candidiasis secondary to the use of TCs is easily treated with nystatin antifungal cream. However, applying nystatin formula $100000 \mathrm{UI} / \mathrm{cc}$ is very effective in preventing this complication. In our opinion, one should always proceed in this manner when anticipating a treatment for more than 10 days, especially when using high strength topical corticosteroids in aqueous solutions $(3,8)$.

Other local adverse effects are burning mouth, hypogeusia, oral hairy leukoplakia and hypersensitive reactions to the drug $(3,12)$.

- Systemic adverse effects

In patients treated with high-potency $\mathrm{TCs}$, there may 
be adverse effects that show the systemic absorption of the drug with inhibition of the hypothalamic-pituitaryadrenal axis and secondary adrenal insufficiency. In our studies, these have been moon face, hirsutism and capillary fragility (4). Such effects have always manifested in a small number of patients. Through the determination of basal plasma cortisol in patients treated with $\mathrm{CP}$ in aqueous solution, we have verified (data not published) the existence of an inhibition of the hypothalamic-pituitary-adrenal axis in $85 \%$ of the cases, occurring in the initial phases of treatment when maximum dosages are used ( 3 rinses/day). This indicates that a secondary adrenal insufficiency is occurring due to an exogenous glucocorticosteroid exposure, which is very often asymptomatic. However, in the maintenance phases, when using very low dosages of the drug in order to maintain the effect of the treatment (one rinse every other day), only $4 \%$ of patients present low levels of plasma cortisol, indicative of inhibition of the axis with adrenal insufficiency. This indicates that usually the secondary adrenal insufficiency to the treatment with high dosages of $\mathrm{CP}$ is reversible when the dosage is reduced; and on the other hand, this reveals that maintenance treatment with low dosages of CP is very safe. However, although there is not an consensus on the subject, it should be taken into consideration that these patients may be at risk for developing an acute adrenal crisis (Addisonian crisis) in the event that, due to stressful circumstances (major surgery, severe traumatism, acute infection, etc.), an increased physiological adrenaline production of corstisol -incapable of reaching the inhibited axis- should be required. This situation may require the exogenous supply of cortisol. We believe this situation to have two implications that are of interest. Patients undergoing treatment of a high strength TC should be subject to evaluations on the evolution of their hypothalamic-pituitary-adrenal axis by means of determining the basal plasma cortisol (levels less than $3 \mu \mathrm{g} / \mathrm{dl}$ are indicative of inhibition) at least once during the onset of the treatment and during the maintenance period. In case of inhibition of the axis, patients and their relatives should be informed of these aspects, and should perhaps carry a letter for the doctor who would have to treat them in the hypothetical case that medical attention should be required for a serious illness. Generally speaking, the usual dental procedures (extraction, curettage, gingivectomy, etc.) do not appear to require an exogenous supply of glucocorticosteroids in patients with inhibition of the hypothalamic-pituitary-adrenal axis (13-15).

\section{References}

1. González-Moles MA, Scully C. Vesiculo-erosive oral mucosal disease management with topical corticosteroids: (1) Fundamental principles and specific agents available. J Dent Res. 2005;84:294-301.

2. González-Moles MA, Scully C. Vesiculo-erosive oral mucosal disease management with topical corticosteroids: (2) Protocols, monitoring of effects and adverse reactions, and the future. J Dent Res. 2005;84:302-8.

3. Lozada-Nur F, Huang MZ, Zhou GA. Open preliminary clinical trial of clobetasol propionate ointment in adhesive paste for treatment of chronic oral vesiculoerosive diseases. Oral Surg Oral Med Oral Pathol. 1991;71:283-7.

4. Gonzalez-Moles MA, Morales P, Rodriguez-Archilla A, Isabel IR, Gonzalez-Moles S. Treatment of severe chronic oral erosive lesions with clobetasol propionate in aqueous solution. Oral Surg Oral Med Oral Pathol Oral Radiol Endod. 2002;93:264-70.

5. Carbone M, Conrotto D, Carrozzo M, Broccoletti R, Gandolfo S, Scully C. Topical corticosteroids in association with miconazole and chlorhexidine in the long-term management of atrophic-erosive oral lichen planus: a placebo-controlled and comparative study between clobetasol and fluocinonide. Oral Dis. 1999;5:44-9.

6. Lozada-Nur F, Miranda C, Maliksi R. Double-blind clinical trial of $0.05 \%$ clobetasol propionate (corrected from proprionate) ointment in orabase and $0.05 \%$ fluocinonide ointment in orabase in the treatment of patients with oral vesiculoerosive diseases. Oral Surg Oral Med Oral Pathol. 1994;77:598-604.

7. Gonzalez-Moles MA, Ruiz-Avila I, Rodriguez-Archilla A, Morales-Garcia P, Mesa-Aguado F, Bascones-Martinez A, et al. Treatment of severe erosive gingival lesions by topical application of clobetasol propionate in custom trays. Oral Surg Oral Med Oral Pathol Oral Radiol Endod. 2003;95:688-92.

8. Lozada-Nur F, Miranda C. Oral lichen planus: topical and systemic therapy. Semin Cutan Med Surg. 1997;16:295-300.

9. González-García A, Diniz-Freitas M, Gándara-Vila P, Blanco-Carrión A, García-García A, Gándara-Rey J. Triamcinolone acetonide mouth rinses for treatment of erosive oral lichen planus: efficacy and risk of fungal over-infection. Oral Dis. 2006;12:559-65.

10. Hegarty AM, Hodgson TA, Lewsey JD, Porter SR. Fluticasone propionate spray and betamethasone sodium phosphate mouthrinse: a randomized crossover study for the treatment of symptomatic oral lichen planus. J Am Acad Dermatol. 2002;47:271-9.

11. Aguirre JM, Bagán JV, Rodriguez C, Jimenez Y, Martínez-Conde R, Díaz de Rojas F, et al. Efficacy of mometasone furoate microemulsion in the treatment of erosive-ulcerative oral lichen planus: pilot study. J Oral Pathol Med. 2004;33:381-5.

12. Bircher AJ, Pelloni F, Langauer Messmer S, Müller D. Delayed hypersensitivity reactions to corticosteroids applied to mucous membranes. Br J Dermatol. 1996;135:310-3.

13. Baid SK, Nieman LK. Therapeutic doses of glucocorticoids: implications for oral medicine. Oral Dis. 2006;12:436-42.

14. Salem M, Tainsh RE Jr, Bromberg J, Loriaux DL, Chernow B. Perioperative glucocorticoid coverage. A reassessment 42 years after emergence of a problem. Ann Surg. 1994;219:416-25.

15. Key SJ, Hodder SC, Davies R, Thomas DW, Thompson S. Perioperative corticosteroid supplementation and dento-alveolar surgery. Dent Update. 2003;30:316-20. 